\title{
Enfermedad inflamatoria intestinal y embarazo: experiencia de 16 años
}

\author{
MARÍA TERESA VERGARA A. ${ }^{1}$, PAULA REY G. ${ }^{2, a}$
}

\section{Pregnancy and inflammatory bowel disease. Experience in 17 patients}

Background: Inflammatory bowel disease (IBD) has a peak incidence between 15 and 25 years of age, thereby affecting women of reproductive age. Fertility rates with inactive IBD are similar to the general population, and drugs currently used, with the exception of methotrexate and thalidomide, have a good safety and efficacy profile during pregnancy. Starting a pregnancy with inactive IBD significantly reduces the potential maternal and fetal complications. Aim: To assess the evolution of pregnancy and the underlying disease in women with IBD. Patients and Methods: Retrospective and prospective study of female patients with IBD controlled in our hospital who became pregnant from January 1994 to February, 2011. Results: We followed 17 patients with a total of 19 pregnancies. In two patients the onset of IBD occurred during pregnancy and from the remaining, 11 patients became pregnant during remission of IBD. Most of the patients continued the same treatment during pregnancy and the few flares that occurred were treated satisfactorily. Major complications occurred in three patients, all associated with IBD activity. Fifteen patients had full-term deliveries and the majority of the newborns had normal weight and Apgar score. None had malformations. Conclusions: Pregnancies among patients with an inactive IBD, have a good evolution. A multidisciplinary approach and patient education are invaluable to achieve these good results.

(Rev Med Chile 2011; 139: 1421-1427).

Key words: Delivery, obstetric; Inflammatory bowel diseases; Pregnancy complications.

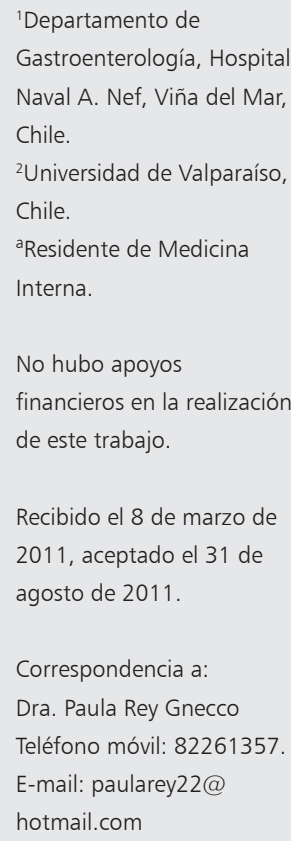

I a enfermedad inflamatoria intestinal (EII) tiene un pico de incidencia entre los 15 y 25 \lrcorner años ${ }^{1}$, afectando por lo tanto a mujeres en edad reproductiva.

La tasa de fertilidad comunicada en la mujer con EII inactiva es similar a la población general ${ }^{2}$. La enfermedad activa, por el contrario, se asocia a una disminución de la fertilidad, la que se ha explicado en la enfermedad de Crohn (EC) por procesos inflamatorios y adherenciales de las trompas de Falopio u ovarios, y una menor frecuencia de relaciones sexuales debido a dispareunia ${ }^{1}$. También se ha descrito aumento de la tasa de infertilidad en pacientes con colitis ulcerosa (CU) tras ser sometidas a proctocolectomía con anastomosis íleo-anal.
Se sugiere como causa la amplia disección a nivel pelviano con riesgo de formación de adherencias que comprometan las trompas de Falopio ${ }^{3}$. En pacientes sometidas a colectomía con anastomosis íleorrectal, cirugía de mayor simplicidad técnica y de baja morbilidad, se ha informado una menor disminución de la tasa de fertilidad ${ }^{3,4}$.

Uno de los avances más significativos durante la última década en el manejo de estas pacientes ha sido la evidencia de que la inactividad al momento de la concepción y durante la gestación es el factor más relevante para lograr un embarazo con resultado perinatal favorable ${ }^{5,6}$. La EII activa en este período se ha asociado a un aumento en la incidencia de abortos, mortinatos, recién nacidos 
de bajo peso, partos de pre-término y cesáreas ${ }^{7,8}$. Las guías de tratamiento vigentes enfatizan la recomendación de planear el embarazo para que se inicie en remisión y continuar durante la gestación con su terapia de mantención, se sostienen las mismas recomendaciones en cuanto a fármacos y dosis que en pacientes no embarazadas.

La mayoría de las drogas utilizadas actualmente en el tratamiento de las EII presentan un buen perfil de seguridad y eficacia durante el embarazo y lactancia ${ }^{10}$, salvo el metotrexato y talidomida, contraindicados por el riesgo de teratogenicidad. Se han comunicado casos aislados de retardo del crecimiento intrauterino, prematuridad e inmunodepresión fetal en pacientes tratadas con azatioprina y 6-mercaptopurina, considerados como fármacos clase D por la FDA. La ciclosporina, clasificada como clase $\mathrm{C}$, se ha asociado a casos de retardo del crecimiento intrauterino y prematuridad ${ }^{11}$. Con respecto al uso de agentes biológicos anti TNF alfa, la mayoría de los estudios a la fecha, realizados con infliximab y adalimumab, muestran resultados perinatales similares a la población general ${ }^{12,13}$.

El objetivo de este estudio fue analizar la evolución del embarazo y el comportamiento de la EII durante éste, en las pacientes portadoras de dicha patología controladas en nuestro centro.

\section{Material y Pacientes}

Se revisaron los embarazos producidos en el grupo de pacientes controladas en el policlínico de EII del Hospital Naval Almirante Nef de Viña del Mar desde enero de 1994 a febrero de 2011. Se realizó un estudio descriptivo, retro y prospectivo de revisión de fichas clínicas recogiendo información sobre el tipo, edad al diagnóstico y extensión de la EII, tratamiento empleado y actividad al momento de la concepción y durante el embarazo, momento y vía del parto, peso, puntaje de Apgar, presencia de malformaciones del recién nacido y complicaciones maternas o fetales durante el embarazo o período perinatal.

\section{Resultados}

Durante el período revisado se registraron 19 embarazos en 17 pacientes.
Las características generales de las pacientes se presentan en la Tabla 1.

De las 17 pacientes de la serie, sólo una presentaba EC. Dos pacientes presentaron el debut de la EII durante el embarazo y dos pacientes con diagnóstico previo de EII fueron derivadas a este centro para continuar sus controles con gestación en curso. Las 13 pacientes restantes se encontraban en control periódico previo al embarazo en el policlínico de EII de nuestro hospital.

Once de los 17 embarazos $(64,7 \%)$ de las pacientes con EII diagnosticada antes del comienzo de la gestación se iniciaron en etapa de remisión de su enfermedad.

La actividad y extensión de la EII junto con el tratamiento empleado al momento de la concepción y durante el embarazo, se presentan en la Tabla 2 y en los Figuras 1, 2 y 3.

Tres pacientes iniciaron su embarazo sin tratamiento debido a suspensión de éste sin indicación médica.

La paciente con EC mantuvo su tratamiento habitual con azatioprina y mesalazina durante el embarazo.

Hubo 3 pacientes que iniciaron la gestación estando en tratamiento esteroidal: una con hepatitis autoinmune y colangitis esclerosante primaria como enfermedad de base que durante la evolución desarrolló una CU. La segunda paciente inició controles en este recinto con embarazo en curso,

Tabla 1. Características generales de las pacientes

\begin{tabular}{ll}
\hline $\begin{array}{l}\text { Edad media al } \\
\text { diagnóstico de Ell }\end{array}$ & 28,4 años (20-37) \\
$\begin{array}{ll}\text { Edad media de } \\
\text { embarazo con Ell }\end{array}$ & 31,5 años (22-42) \\
$\begin{array}{l}\text { Colitis ulcerosa } \\
\text { idiopática }\end{array}$ & 16 pacientes: \\
& • Proctitis:4 \\
& - Colitis izquierda: 5 \\
& - Colitis extensa:5 \\
& • Extensión no determinada: 2* \\
& 1 paciente: \\
Enfermedad de Crohn & - lleon-colon
\end{tabular}

*antecedente de colitis izquierda en fibrosigmoidoscopia previa, se encuentra pendiente colonoscopia completa con biopsia escalonada (cursando puerperio tardío). 
Tabla 2: Actividad de la enfermedad por paciente, al momento del inicio del embarazo y su evolución

\begin{tabular}{|c|c|c|c|}
\hline Pac. & Diagnóstico & Concepción & Curso de embarazo \\
\hline 1 & CU & Debut CU durante el embarazo & $\begin{array}{l}\text { Activa severa. Colectomía subtotal en puer- } \\
\text { perio precoz }\end{array}$ \\
\hline 2 & CU & Debut CU durante el embarazo & Se logra control de enfermedad \\
\hline 3 & CU & Actividad moderada. Suspendió tratamiento & $\begin{array}{l}\text { Aborto } 20 \text { sem. Corioamnionitis + hematoma } \\
\text { retroplacentario }\end{array}$ \\
\hline 4 & $\mathrm{CU}$ & Actividad moderada. Suspendió tratamiento & Se logra control de enfermedad \\
\hline 5 & CU & Actividad moderada & Actividad leve \\
\hline \multirow[t]{2}{*}{6} & CU & $1^{\circ}$ embarazo: A. leve & Aborto, genopatía trisomia 16 \\
\hline & & $2^{\circ}$ embarazo: A. leve & $2^{\circ}$ embarazo: Se logra control de enfermedad \\
\hline \multirow[t]{2}{*}{7} & CU & $1^{\circ}$ embarazo: $A$. leve & $1^{\circ}$ embarazo: Se logra control de enfermedad \\
\hline & & $2^{\circ}$ embarazo: Remisión & $2^{\circ}$ embarazo: Remisión \\
\hline 8 & EC & Remisión & Reactivación leve $3^{\circ}$ trimestre \\
\hline 9 & CU & Remisión & Reactivación leve $1^{\circ}$ trimestre \\
\hline 10 & CU & Remisión & Remisión \\
\hline 11 & CU & Remisión & Remisión \\
\hline 12 & CU & Remisión & Remisión \\
\hline 13 & CU & Remisión & Remisión \\
\hline 14 & CU & Remisión & Remisión \\
\hline 15 & $\mathrm{CU}$ & Remisión & Remisión \\
\hline 16 & $\mathrm{CU}$ & Remisión & Remisión \\
\hline 17 & $\mathrm{CU}$ & Remisión & Remisión \\
\hline
\end{tabular}

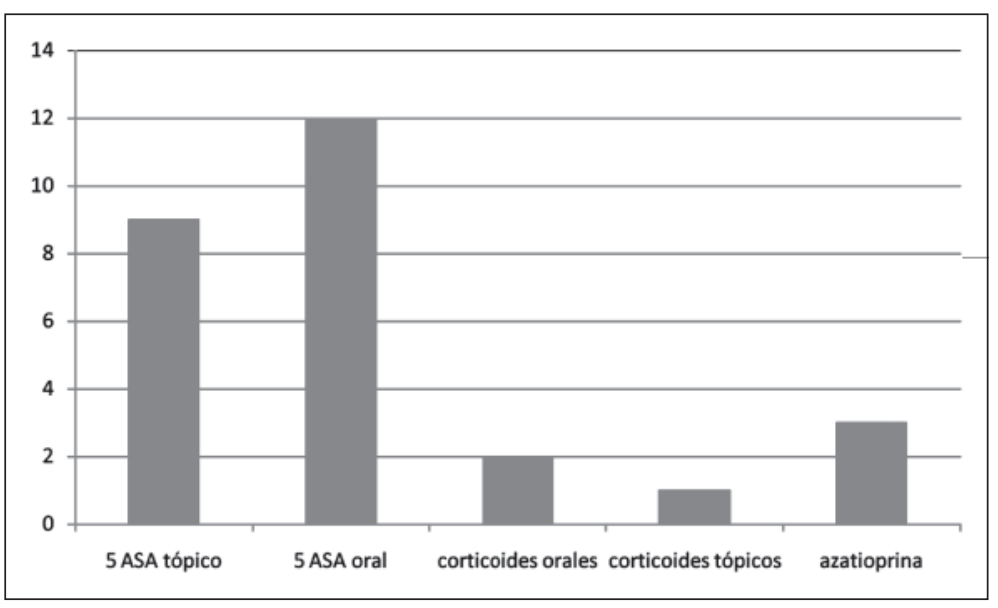

Figura 1. Tratamiento al inicio del embarazo de pacientes con CU. 


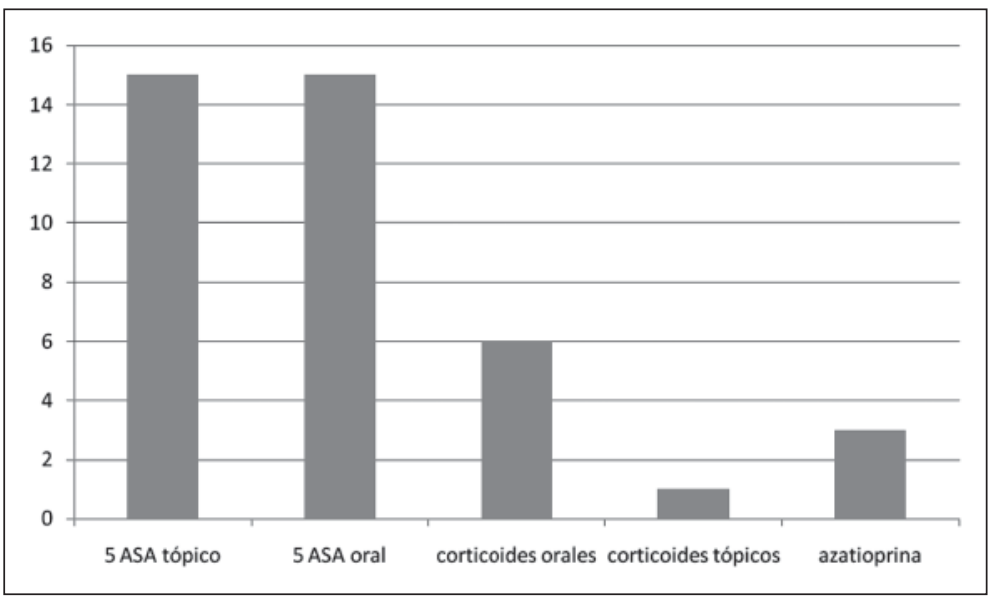

Figura 2. Tratamiento durante el embarazo de pacientes con CU. en remisión y en tratamiento con budesonida y 5- aminosalicilatos (5-ASA) oral por una CU cuya extensión no estaba claramente determinada, la única colonoscopia realizada había evaluado hasta el sigmoides mostrando un compromiso difuso hasta esa área. Se realizó cambió a 5-ASA exclusivo, oral y tópico. La tercera paciente, portadora de CU extensa, se manejó con 5-ASA oral asociado a tratamiento con enemas de hidrocortisona debido a falta de medios económicos para adquirir 5-ASA tópicos. Las tres pacientes se mantuvieron estables, sin complicaciones durante el embarazo.

Durante la gestación, además de las pacientes descritas, 5 recibieron tratamiento esteroidal, correspondiendo a las dos pacientes que debutaron con la EII durante el embarazo, una que se embarazó activa debido a suspensión de tratamiento y 2 pacientes con reactivaciones durante el primer y tercer trimestre de embarazo respectivamente. De estas pacientes, 2 presentaron complicaciones mayores: una que debutó con una CU extensa durante el embarazo y a pesar del tratamiento con esteroides sistémicos y 5 ASA se mantuvo activa. Evolucionó con una anemización severa y rápidamente progresiva, que terminó en la interrupción de la gestación mediante cesárea a las $32+5$ semanas. La mala evolución de la paciente no permitió intentar tratamiento con inmunomoduladores como azatioprina o 6 mercaptopurina ya que existe una latencia de 12 a 16 semanas entre la instauración del tratamiento y el inicio de su efecto, $y$ en esa época no se disponía de agentes biológicos. La paciente presentó en el puerperio precoz una hemorragia digestiva baja masiva con compromiso hemodinámico, requiriendo una colectomía de urgencia. La segunda paciente se embarazó activa debido a suspensión de tratamiento. Requirió tratamiento con esteroides sistémicos con respuesta favorable inicial, presentando una reactivación al intentar su retiro. Se mantuvo una dosis de 12,5 mg de prednisona hasta la semana 37 , en que se interrumpió el embarazo mediante cesárea por feto con retardo del crecimiento intrauterino (RCIU). No se pudo realizar tratamiento con tiopurínicos por la misma razón que en la paciente descrita previamente. En cuanto al uso de agentes biológicos no se pudo acceder por falta de medios económicos.

La única paciente de la serie que recibió estos agentes fue una portadora de una proctitis ulcerosa, bajo tratamiento local con 5 ASA, en remisión al momento de la concepción, que recibió 6 dosis de etanercept (antiTNF alfa) como agente biológico de segunda línea debido a una espondiloartropatía seronegativa que no respondió a infliximab. El fármaco había sido suspendido por una neumonía grave semanas antes que se confirmara el embarazo. La paciente evolucionó asintómatica durante toda la gestación y fue sometida a cesárea electiva por su sacroileitis. El recién nacido no presentó complicaciones.

En la Tabla 3 se describe la duración del embarazo y la vía de parto, junto con el peso y puntaje de Apgar de los recién nacidos. No hubo malformaciones en los recién nacidos de la serie. Quince de los 17 partos fueron de término. En este 
Tabla 3. Caracterización de los embarazos, partos y recién nacidos

\begin{tabular}{|c|c|c|c|}
\hline Embarazos & Parto & Peso RN promedio & Apgar $\left(1^{\prime}-5^{\prime}\right)$ \\
\hline \multirow[t]{3}{*}{ Término: 15} & $\operatorname{PTVE}\left({ }^{*}\right): 7$ & $3.423 \mathrm{~g}(3.120-3.940 \mathrm{gr})$ & Normal \\
\hline & Fórceps: 1 & $3.090 \mathrm{~g}$ & Normal \\
\hline & Cesárea:7 & $3.397 \mathrm{~g}(2.440-3.980 \mathrm{gr})$ & 6 Normales, 1 RN con Apgar 5-8 \\
\hline \multirow[t]{2}{*}{ Pre término: 2} & Cesárea: 1 & $1.450 \mathrm{~g}$ & Normal \\
\hline & $\operatorname{PPTVE}(\dagger): 1$ & $3.030 \mathrm{~g}$ & Normal \\
\hline Abortos: 2 & \multicolumn{3}{|c|}{$\begin{array}{c}\text { Corioamnionitis aguda }+ \text { hematoma retroplacentario } \\
\text { Trisomía } 16\end{array}$} \\
\hline
\end{tabular}

RN: recién nacido, *PTVE: parto de término, de vértice, espontáneo; (†) PPTVE: parto de pre-término, de vértice, espontáneo.

grupo se realizaron 7 cesáreas, sólo una motivada por actividad de la EII.

El peso promedio y los puntajes de Apgar al minuto y cinco minutos de los recién nacidos de término fueron normales, salvo el con RCIU previamente descrito, que pesó $2.440 \mathrm{gr}$ con puntaje de Apgar 5-8.

Hubo 2 partos de pretérmino. Uno de $36+4$ semanas de gestación, vaginal espontáneo, con producto de 3.030 gramos y Apgar normal, sin complicaciones. El segundo correspondió a una de las pacientes que debutó con CU en el embarazo, que presentó mala evolución, determinándose la interrupción del embarazo mediante cesárea a las $32+5$ semanas. El recién nacido pesó 1.450 gramos, con Apgar normal.

Se produjeron 2 abortos espontáneos, uno durante el primer trimestre en una paciente con CU inactiva, secundario a una genopatía (trisomía del cromosoma 16) y otro debido a una corioamnionitis aguda con hematoma retroplacentario, que correspondió a una paciente con CU extensa que suspendió tratamiento e inició su gestación con enfermedad activa. Al momento del aborto se encontraba con la EII en remisión, bajo tratamiento con 5-ASA oral y tópico.

Las complicaciones durante la gestación no relacionadas con la EII fueron un caso de síndrome hipertensivo del embarazo, otra paciente que presentó diabetes gestacional, quien además tuvo una pielonefritis aguda y una paciente con varicela con compromiso cutáneo exclusivo. De estas, sólo la paciente que presentó varicela estaba bajo tratamiento inmunosupresor (azatioprina por EC), ninguna estaba con tratamiento esteroidal. No hubo complicaciones severas de estas patologías.

\section{Discusión}

En la literatura en general existe escasa información en relación a la EII y embarazo. En nuestro medio, además de una serie presentada recientemente en un congreso de la especialidad ${ }^{14}$, la nuestra a pesar de su tamaño limitado, corresponde a la más amplia publicada a nivel nacional. Los resultados perinatales del grupo han sido satisfactorios, presentando 15 de 17 (88\%) partos de término y 15 de $17(88 \%)$ recién nacidos con peso sobre $3.000 \mathrm{~g}$.

La mayoría de los embarazos se iniciaron en remisión, permaneciendo las pacientes inactivas, en gran parte, con el mismo esquema y dosis de tratamiento en uso previo a la concepción. Las escasas reactivaciones presentadas fueron tratadas exitosamente y las complicaciones de mayor gravedad ocurrieron en pacientes con EII activa. No se registraron malformaciones ni otras complicaciones que pudiéramos atribuir al tratamiento empleado. Nuestros resultados son concordantes con lo descrito en la literatura.

Con respecto a los 2 abortos espontáneos registrados en nuestra serie, uno relacionado a corioamnionitis y el segundo a trisomía del cromosoma 16, encontramos una publicación que informa una incidencia mayor de corioamionitis en embarazadas con EII versus controles ${ }^{5}$. Sin embargo, no se describe en este trabajo si se analizó la existencia de una relación con el tipo de enfermedad, actividad o el tratamiento empleado. En nuestro caso, la paciente había comenzado el embarazo con una colitis extensa activa, pero al momento del aborto se encontraba en remisión y sin uso de fármacos inmunosupresores. Por otro 
lado, la presencia de trisomía del cromosoma 16, que lleva en la mayoría de los casos a aborto durante el primer trimestre ${ }^{15}$, corresponde a una alteración presente hasta en $1 \%$ de la población general embarazada y no se ha descrito en la literatura ninguna asociación con la EII.

Otras complicaciones del embarazo, como síndrome hipertensivo y diabetes gestacional, no presentaron una incidencia mayor a la reportada en la población general ${ }^{16,17}$.

En relación a estos antecedentes, creemos que un punto importante a analizar es que a pesar los resultados satisfactorios publicados durante los últimos años gracias al desarrollo de terapias que han demostrado ser eficaces y seguras, las pacientes con EII presentan tasas de embarazo significativamente menores que la población general, aun con enfermedad inactiva ${ }^{1}$. Encuestas realizadas a los pacientes han mostrado que un factor importante constituye la decisión voluntaria de no concebir, producto del temor a eventuales efectos teratogénicos de los fármacos, a complicaciones del embarazo y al riesgo de transmitir la enfermedad a la descendencia ${ }^{18,19}$.

Nos parece por lo tanto, que la educación de las pacientes con EII en edad fértil debe ser un tema fundamental en su manejo. Para ello, el grupo de profesionales tratantes que incluye gastroenterólogos, gineco-obstétras, cirujanos, nutriólogos, etc, deben estar interiorizados en el tema para entregar la información necesaria que permita superar las posibles aprensiones asociadas a la maternidad y a la EII, así como también ante un embarazo en curso, proseguir con el tratamiento ya instaurado y los controles adecuados.

A nuestro parecer, las recomendaciones más importantes de recalcar son las ventajas de programar el embarazo para que se inicie en remisión, la seguridad de los fármacos usados actualmente en el tratamiento (con la excepción de metotrexato y talidomida), y el riesgo mayor que implica su suspensión.

A pesar de la menor experiencia con agentes anti TNF $\alpha$, se ha acumulado a nivel internacional una casuística significativa en pacientes con EII embarazadas bajo tratamiento principalmente con infliximab y adalimumab, con resultados perinatales similares a la población general, lo que permite considerarlos como seguros ${ }^{12,13}$.

Otro punto a mencionar es el aumento de la tasa de infertilidad de hasta 3 veces descrito en pa- cientes CU tras ser sometidas a proctocolectomía con anastomosis íleo-anal ${ }^{3}$. En pacientes jóvenes con EII que puedan buscar posteriormente un embarazo debe tenerse en cuenta este antecedente, y ante la indicación de cirugía evaluar la opción de una colectomía con anastomosis íleorrectal, procedimiento con el que se ha descrito menor repercusión en la tasa de fertilidad ${ }^{3,4}$ al ser de mayor simplicidad técnica y con menor riesgo de formación de adherencias. Esto deja la posibilidad de manejar la actividad de la enfermedad a nivel rectal con terapia médica local o en forma quirúrgica en un tiempo futuro.

Creemos que un manejo multidisciplinario adecuado y un seguimiento cercano de la paciente durante el embarazo permitirá el tratamiento pre$\mathrm{COz}$ de eventuales reactivaciones y finalmente ante la pregunta, ¿cuál debe ser la vía del parto?, debe recomendarse la vía vaginal si es que no existe enfermedad perianal activa u otra contraindicación de tipo obstétrica para ella ${ }^{1,2}$.

\section{Referencias}

1. Habal FM, Kapila V. Inflammatory bowel disease and pregnancy: evidence, uncertainty and patient decisionmaking. Can J Gastroenterol 2009; 23 (1): 49-53.

2. Steinlauf AF, Present DH. Medical management of the pregnant patient with inflammatory bowel disease. Gastroenterol Clin North Am 2004; 33: 361-85.

3. Waljee A, Waljee J, Morris AM, Higgins P. Threefold increased risk of infertility: a meta-analysis of infertility after ileal pouch anal anastomosis in ulcerative colitis. Gut 2006; 55: 1575-80.

4. Bellolio F, Zúñiga J, Wagner P, Pinedo G, Duarte I, Zúñiga A. Anastomosis ileorrectal en el tratamiento quirúrgico de la colitis ulcerosa: Resultados a largo plazo, Rev Med Chile 2008; 136: 1121-6.

5. Bush MC, Patel S, Lapinski RH, Stone JL. Perinatal outcomes in inflammatory bowel disease. J Matern Fetal Neonatal Med 2004; 15: 237-41.

6. Mottet C, Juillerat P, Gonvers J, Froehlich F, Burnand B, Vader J, et al. Pregnancy and Crohn's disease.Digestion 2005; 71: 54-61.

7. Subhani JM, Hamilton MI. Review article: The management of inflammatory bowel disease during pregnancy. Aliment Pharmacol Ther 1998; 12: 1039-53.

8. Cornish J, Tan E, Teare J, Teoh TG, Rai R, Clark SK, et al. A meta-analysis on the influence of inflammatory bowel disease on pregnancy. Gut 2007; 56: 830-7. 
9. Carter MJ, Lobo AJ, Travis SPL. British Society of Gastroenterology. Guidelines for the management of inflammatory bowel disease in adults. Gut 2004; 53 (Suppl V): V1-16

10. Moskovitz DN, Bodian C, Chapman ML, Marion JF, Rubin PH, Scherl E, et al. The effect on the fetus of medications used to treat pregnant inflammatory bowel disease patients. Am J Gastroenterol 2004; 656-61.

11. Correia LM, Bonilha DQ, Ramos JD, Ambrogini O, Miszputen SJ. Treatment of inflammatory bowel disease and pregnancy: a review of the literature. Arq Gastroenterol 2010; 47 (2): 197-201.

12. Katz JA, Antoni C, Keenan GF, Smith DE, Jacobs SJ, Lichtenstein GR. Outcome of pregnancy in women receiving infliximab for the treatment of Crohn's disease and rheumatoid arthritis. Am J Gastroenterol 2004; 99: 2385-92.

13. Mahadevan U, Kane S, Sandborn WJ, Cohen RD, Hanson K, Terdiman JP, Binion DG. Intentional infliximab use during pregnancy for induction or maintenance of remission in Crohn's disease. Aliment Pharmacol Ther
2005; 21: 733-8.

14. Figueroa C, Quera R. Embarazo no influye en la evolución de la enfermedad inflamatoria intestinal. (presentado en el Congreso Chileno de Gastroenterología 2009). Gastroenterología Latinoamericana 2009; 20 (3).

15. Arakaki DT, Waxman SH. Chromosome abnormalities in early spontaneous abortions J Med Genet 1970; 7: 118-24

16. Duley L. The global impact of preeclampsia and eclampsia. Semin Perinatol 2009; 33 (3): 130-7.

17. Huidobro A, Fulford A, Carrasco E. Incidencia de diabetes gestacional y su relación con obesidad en embarazadas chilenas. Rev Med Chile 2004; 132: 931-8.

18. Mountifield R, Bampton P, Prosser R, Muller K, Andrews J. Fear and Fertility in Inflammatory Bowel Disease: A Mismatch of Perception and Reality Affects Family Planning Decisions. Inflamm Bowel Dis 2009; 15: 720-5.

19. Reddy S, Wolf J. Management issues in women with inflammatory bowel disease. J Am Osteop Ass 2001; 101: S17-2. 